Int. J. Contemp. Math. Sciences, Vol. 2, 2007, no. 27, 1307 - 1316

\title{
Approximate Solutions for Nonlinear Fractional Heat Equations
}

\author{
Mahmoud M. El-Borai ${ }^{1}$, Khairia El-Said El-Nadi \\ and Eman G. EL-Akabawy \\ Faculty of Science \\ Alexandria University \\ Alexandria, Egypt
}

\begin{abstract}
In this paper, we shall study the approximate solutions of the Cauchy problem,

$$
\begin{gathered}
\left(D_{0^{+}}^{\alpha} u\right)(x, t)=\sum_{i=1}^{n} \frac{\partial^{2}(x, t)}{\partial x_{i}^{2}}+f(x, t, u(x, t)), \\
u(x, 0)=\varphi(x),
\end{gathered}
$$

where $0<\alpha<1$, and $f(x, t, u)$ is a continuous function of the three variables $\mathrm{x}, \mathrm{t}$ and $\mathrm{u}$ on the domain

$$
Q=\left\{(x, t, u): x \in R^{n}, 0 \leq t \leq h,|u-\varphi| \leq \mu, \mu>0\right\} .
$$

Also, some properties of the solutions of the considered problem are established.
\end{abstract}

Mathematical Subject Classification: 26A33, 45D05, 45N05

Keywords: Nonlinear fractional heat equations, approximate solutions

\section{Introduction:}

In this paper we consider the approximate solutions of the problem

$$
\left(D_{0^{+}}^{\alpha} u\right)(x, t)=\sum_{i=1}^{n} \frac{\partial^{2} u(x, t)}{\partial x_{i}^{2}}+f(x, t, u(x, t)),
$$

\footnotetext{
${ }^{1}$ m_m_elborai@yahoo.com
} 


$$
u(x, 0)=\varphi(x)
$$

with $0<\alpha<1$ over the interval [0,a], [1,2].

Suppose that $f(x, t, u)$ is continuous on the domain $\mathrm{Q}$.

Thus

$$
\max _{(x, t, u) \in Q}|f(x, t, u)|=M<\infty .
$$

According to [3], the Cauchy problems (1.1), (1.2) are equivalent to the integral equation

$$
\begin{gathered}
u(x, t)=\int_{0}^{\infty} \int_{R_{n}} \zeta_{\alpha}(\theta) G\left(x-\xi, t^{\alpha} \theta\right) \varphi(\xi) d \xi d \theta \\
+\alpha \int_{0}^{t} \int_{0}^{\infty} \int_{R_{n}} \theta(t-\eta)^{\alpha-1} \xi_{\alpha}(\theta) G\left(x-\xi,(t-\eta)^{\alpha} \theta\right) f(\xi, \eta, u(\xi, \eta)) d \xi d \theta d \eta .
\end{gathered}
$$

where $\zeta_{\alpha}(\theta)$ is a probability density function defined on $(0, \infty)$ and

$$
G(x, t)=\frac{e^{-|x|^{2} / 4 t}}{(\sqrt{4 \pi t})^{n}}, \quad|x|^{2}=x_{1}^{2}+x_{2}^{2}+\ldots \ldots+x_{n}^{2},[4,5] .
$$

\section{Approximate solutions:}

To construct the approximate solutions of (1.4) let us choose $u_{1}(x, t)$ by $[6]$

$$
\begin{gathered}
u_{1}(x, t)=\int_{0}^{\infty} \int_{R^{n}} \zeta_{\alpha}(\theta) G\left(x-\xi, t^{\alpha} \theta\right) \varphi(\xi) d \xi d \theta \\
+\alpha \int_{0}^{t} \int_{0}^{\infty} \int_{R^{n}} \theta(t-\eta)^{\alpha-1} \zeta_{\alpha}(\theta) G\left(x-\xi,(t-\eta)^{\alpha} \theta\right) f\left(\xi, \eta, \alpha_{1}(\xi)\right) d \xi d \theta d \eta .
\end{gathered}
$$

and

$$
\alpha_{1}(x)=\frac{1}{h} \int_{0}^{h} u_{1}(x, t) d t .
$$

From (1.5) and (1.6) we get

$$
\begin{gathered}
h \alpha_{1}(x)=\int_{0}^{h} \int_{0}^{\infty} \int_{R^{n}} \zeta_{\alpha}(\theta) G\left(x-\xi, t^{\alpha} \theta\right) \varphi(\xi) d \theta d t \\
+\alpha \int_{0}^{h} \int_{0}^{t} \int_{0}^{\infty} \int_{R^{n}} \theta(t-\eta)^{\alpha-1} \zeta_{\alpha}(\theta) G\left(x-\xi,(t-\eta)^{\alpha} \theta\right) f\left(\xi, \eta, \alpha_{1}(\xi)\right) d \xi d \theta d \eta .
\end{gathered}
$$

For the second approximation let us write

$$
\begin{gathered}
u_{2}(x, t)=\int_{0}^{\infty} \int_{R^{n}} \zeta_{\alpha}(\theta) G\left(x-\xi, t^{\alpha} \theta\right) \varphi(\xi) d \xi d \theta \\
+\alpha \int_{0}^{t} \int_{0}^{\infty} \int_{R^{n}} \theta(t-\eta)^{\alpha-1} \zeta_{\alpha}(\theta) G\left(x-\xi,(t-\eta)^{\alpha} \theta\right) f\left(\xi, \eta, u_{1}+\alpha_{2}\right) d \xi d \theta d \eta
\end{gathered}
$$




$$
\alpha_{2}(x)=\frac{1}{h} \int_{0}^{h} \delta_{2}(x, t) d t, \delta_{2}(x, t)=u_{2}(x, t)-u_{1}(x, t) .
$$

According to (1.5) and (1.8) we get

$$
\begin{gathered}
\delta_{2}(x, t)=\alpha \int_{0}^{t} \int_{0}^{\infty} \int_{R^{n}} \theta(t-\eta)^{\alpha-1} \zeta_{\alpha}(\theta) G\left(x-\xi,(t-\eta)^{\alpha} \theta\right)\left[f\left(\xi, \eta, u_{1}+\alpha_{2}\right)\right. \\
\left.-f\left(\xi, \eta, \alpha_{1}\right)\right] d \xi d \theta d \eta
\end{gathered}
$$

and so, according to formula (1.9) we have the following equation

$$
\begin{gathered}
h \alpha_{2}(x)=\alpha \int_{0}^{h} \int_{0}^{t} \int_{0}^{\infty} \int_{R^{n}} \theta(t-\eta)^{\alpha-1} \xi_{\alpha}(\theta) G\left(x-\xi,(t-\eta)^{\alpha} \theta\right)\left[f\left(\xi, \eta, u_{1}+\alpha_{2}\right)\right. \\
\left.-f\left(\xi, \eta, \alpha_{1}\right)\right] d \xi d \theta d \eta d t .
\end{gathered}
$$

continuing this process $\mathrm{m}$ times, we get

$$
\begin{gathered}
u_{m}(x, t)=\int_{0}^{\infty} \int_{R^{n}} \zeta_{\alpha}(\theta) G\left(x-\xi, t^{\alpha} \theta\right) \varphi(\xi) d \xi d \theta \\
+\alpha \int_{0}^{t} \int_{0}^{\infty} \int_{R^{n}} \theta(t-\eta)^{\alpha-1} \xi_{\alpha}(\theta) G\left(x-\xi,(t-\eta)^{\alpha} \theta\right) f\left(\xi, \eta, u_{m-1}+\alpha_{m}\right) d \xi d \theta d \eta \\
(m=3,4, \ldots \ldots)
\end{gathered}
$$

where

$$
\alpha_{m}(x)=\frac{1}{h} \int_{0}^{h} \delta_{m}(x, t) d t, \delta_{m}(x, t)=u_{m}(x, t)-u_{m-1}(x, 1) .
$$

and so from (1.13)

$$
\begin{gathered}
\delta_{m}(x, t)=\alpha \int_{0}^{t} \int_{0}^{\infty} \int_{R^{n}} \theta(t-\eta)^{\alpha-1} \xi_{\alpha}(\theta) G\left(x-\xi,(t-\eta)^{\alpha} \theta\right)\left[f\left(\xi, \eta, v_{m}\right)\right. \\
-f\left(\xi, \eta, v_{m-1}\right] d \xi d \theta d \eta
\end{gathered}
$$

where

$$
v_{m}=u_{m-1}+\alpha_{m} .
$$

Then $\alpha_{m}(x)$ will be defined by the equation

$$
\begin{gathered}
h \alpha_{m}(x)=\alpha \int_{0}^{h} \int_{0}^{t} \int_{0}^{\infty} \int_{R^{n}} \theta(t-\eta)^{\alpha-1} \xi_{\alpha}(\theta) G\left(x-\xi,(t-\eta)^{\alpha} \theta\right)\left[f\left(\xi, \eta, v_{m}\right)\right. \\
-f\left(\xi, \eta, v_{m-1}\right] d \xi d \theta d \eta d t
\end{gathered}
$$

Suppose that $f(x, t, u))$ satisfies the Lipschitz condition with respect to $\mathrm{u}$ :

$$
|f(x, t, u)-f(x, t, v)| \leq L|u-v|, \quad L>0 .
$$




\section{Theorem (1.1):}

Suppose $0<\alpha<1, f(x, t, u(x, t))$ is continuous function on domain

$$
Q=\left\{(x, t, u): x \in R^{n}, 0 \leq t \leq h,|u-\varphi| \leq \mu, \mu>0\right\},
$$

and suppose

$$
\begin{gathered}
\frac{L h^{\alpha}}{\alpha+1}<1, \\
M h^{\alpha}+2 \lambda+\frac{2 M h^{\alpha}}{\alpha+1} \leq \mu,|\varphi(x)| \leq \lambda .
\end{gathered}
$$

Then equation (1.7) has a unique real root $\alpha_{1}(x)$ satisfies the condition

$$
\left|\alpha_{1}(x)-\varphi(x)\right|<\mu .
$$

In addition equation (1.15) has a unique real root $\alpha_{m}, m=2,3, \ldots$ satisfies the condition

$$
\left|\alpha_{m}(x)\right| \leq \frac{2 M h^{\alpha}}{\alpha+1}
$$

and so

$$
\left|u_{m-1}(x, t)+\alpha_{m}(x)-\phi(x)\right| \leq \mu .
$$

proof:

Let us choose the notations

$$
\begin{gathered}
\theta(c(x))=c(x)-\frac{1}{h} \int_{0}^{h} \int_{0}^{\infty} \int_{R^{n}} \zeta_{\alpha}(\theta) G\left(x-\xi, t^{\alpha} \theta\right) \varphi(\xi) d \xi d \theta d t \\
-\frac{\alpha}{h} \int_{0}^{h} \int_{0}^{t} \int_{0}^{\infty} \int_{R^{n}} \theta(t-\eta)^{\alpha-1} \zeta_{\alpha}(\theta) G\left(x-\xi,(t-\eta)^{\alpha} \theta\right) f(\xi, \eta, c) d \xi d \theta d \eta d t \\
\psi_{m}(c(x))=c(x) \\
-\frac{\alpha}{h} \int_{0}^{h} \int_{0}^{t} \int_{0}^{\infty} \int_{R^{n}} \theta(t-\eta)^{\alpha-1} \zeta_{\alpha}(\theta) G\left(x-\xi,(t-\eta)^{\alpha} \theta\right)\left[f\left(\xi, \eta, u_{m-1}+c\right)\right. \\
\left.-f\left(\xi, \eta, u_{m-2}+\alpha_{m-1}\right)\right] d \xi d \theta d \eta d t \\
m=2,3, \ldots \ldots
\end{gathered}
$$

From (1.3) and (1.18), we get

$$
\begin{gathered}
\theta(-\mu+\varphi(x)) \leq-\mu+\varphi+\lambda+\frac{M h^{\alpha}}{\alpha+1} \leq 0, \\
\theta(\mu+\varphi(x)) \geq \mu+\varphi-\lambda-\frac{M h^{\alpha}}{\alpha+1} \geq 0 .
\end{gathered}
$$


This means that the equation $\theta(c)=0$ has root $\alpha_{1}(x)$ satisfies the condition (1.19).We will see that on the interval $[-\mu+\varphi(x), \mu+\varphi(x)]$ this root is unique. Suppose that there is another root $\alpha_{1}^{\prime}(x)$, then

$$
\begin{gathered}
\left|\alpha_{1}-\alpha_{1}\right|=\frac{\alpha}{h} \mid \int_{0}^{h} \int_{0}^{t} \int_{0}^{\infty} \int_{R^{n}} \theta(t-\eta)^{\alpha-1} \xi_{\alpha}(\theta) G\left(x-\xi,(t-\eta)^{\alpha} \theta\right)\left[f\left(\xi, \eta, \alpha_{1}^{1}\right)\right. \\
\left.-f\left(\xi, \eta, \alpha_{1}\right)\right] d \xi d \theta d \eta d t \mid \\
\leq \frac{L h^{\alpha}}{\alpha+1}\left|\alpha_{1}-\alpha_{1}\right|
\end{gathered}
$$

and so $\frac{L h^{\alpha}}{\alpha+1} \geq 1$ which contradicts the inequality (1.17).

According to (1.5) and (1.3), $\left|u_{1}(x, t)-\varphi(x)\right| \leq 2 \lambda+M h^{\alpha}$

then

$$
\varphi(x)-2 \lambda-M h^{\alpha} \leq u_{1}(x, t) \leq \varphi(x)+M h^{\alpha}+2 \lambda
$$

From inequality (1.18)

$$
M h^{\alpha} \leq \mu-2 \lambda-\frac{2 M h^{\alpha}}{\alpha+1}
$$

From (1.21) and (1.22) we get

$$
\begin{gathered}
\varphi(x)-\mu+\frac{2 M h^{\alpha}}{\alpha+1} \leq u_{1}(x, t) \leq \varphi(x)+\mu-\frac{2 M h^{\alpha}}{\alpha+1}, \\
\varphi(x)-\mu \leq u_{1}(x, t)+\left|\frac{2 M h^{\alpha}}{\alpha+1}\right| \leq \varphi(x)+\mu .
\end{gathered}
$$

In addition

$$
\begin{gathered}
\psi_{2}\left(\frac{-2 M h^{\alpha}}{\alpha+1}\right)=\frac{-2 M h^{\alpha}}{\alpha+1} \\
-\frac{\alpha}{h} \int_{0}^{h} \int_{0}^{t} \int_{0}^{\infty} \int_{R^{n}} \theta(t-\eta)^{\alpha-1} \zeta_{\alpha}(\theta) G\left(x-\xi,(t-\eta)^{\alpha} \theta\right)\left[f\left(\xi, \eta, u_{2}-\frac{2 M h^{\alpha}}{\alpha+1}\right)\right. \\
\left.-f\left(\xi, \eta, \alpha_{1}\right)\right] d \xi d \theta d \eta d t \\
\leq \frac{-2 M h^{\alpha}}{\alpha+1}-\frac{2 M h^{\alpha}}{\alpha+1} \leq 0 .
\end{gathered}
$$

Similarly

$$
\begin{aligned}
& \psi_{2}\left(\frac{2 M h^{\alpha}}{\alpha+1}\right) \geq 0 \text { and so equation } \\
& \psi_{2}(c)=0 \text { has a root } \alpha_{2}(x) \in R
\end{aligned}
$$

satisfies the condition

$$
\left|\alpha_{2}(x)\right| \leq \frac{2 M h^{\alpha}}{\alpha+1}
$$


And so $\left|u_{1}(x, t)+\alpha_{2}(x)-\varphi(x)\right| \leq M$

In a similar manner, it is easy to see that on the interval $\left[\frac{-2 M h^{\alpha}}{\alpha+1}, \frac{2 M h^{\alpha}}{\alpha+1}\right]$, the root $\alpha_{2}(x)$ is unique

In general

$$
\begin{gathered}
\left|u_{m-2}(x, t)+\alpha_{m-1}(x)-\varphi(x)\right| \leq M \quad(m=3,4, \ldots . .), \\
\left|u_{m-1}(x, t)-\varphi(x)\right| \leq \frac{M h^{\alpha}}{\alpha+1} \\
-\mu+\varphi(x) \leq u_{m-1}(x, t)+\left|\frac{2 M h^{\alpha}}{\alpha+1}\right| \leq \mu+\varphi(x)
\end{gathered}
$$

and so there exist a root $\alpha_{m}(x) \in R$ of the equation $\psi_{m}(c)=0$ which satisfies the condition

$$
\begin{gathered}
\left|\alpha_{m}(x)\right| \leq \frac{2 M h^{\alpha}}{\alpha+1}, \text { and so } \\
\left|u_{m-1}(x, t)+\alpha_{m}(x)-\varphi(x)\right| \leq \mu .
\end{gathered}
$$

Hence the required result.

Suppose on the interval $[0, \mathrm{~h}]$

$$
\begin{gathered}
\left|u_{1}(x, t)-\varphi(x)\right| \leq \delta,(\delta>0) . \\
\varepsilon=\frac{L h^{\alpha}(\alpha+2)}{(\alpha+1)-L h^{\alpha}} .
\end{gathered}
$$

\section{Theorem 1.2:}

Suppose $0<\alpha<1, f(x, t, u(x, t))$ satisfies the condition of theorem (1.1), the inequalities (1.17) and (1.24) are satisfies, then the sequence $\left\{u_{m}(x, t)\right\}$ which constructed by the previous method uniformly converges on the interval $[0, \mathrm{~h}]$ to a function $\mathrm{u}(\mathrm{x}, \mathrm{t})$ which is the solution of the equation (1.4).

proof:

From (1.11), (1.16) and (1.23) we get

$$
\begin{gathered}
\left|\alpha_{2}(x)\right| \\
\leq \frac{\alpha L}{h} \int_{0}^{h} \int_{0}^{t} \int_{0}^{\infty} \int_{R^{n}} \theta(t-\eta)^{\alpha-1} \zeta_{\alpha}(\theta) G\left(x-\xi,(t-\eta)^{\alpha} \theta\right)\left|u_{1}(x, t)+\alpha_{2}(x)-\alpha_{1}(x)\right| d \xi d \theta d \eta d t \\
\leq \frac{\alpha L\left|\alpha_{2}(x)\right|}{h} \int_{0}^{h} \int_{0}^{t} \int_{0}^{\infty} \int_{R^{n}} \theta(t-\eta)^{\alpha-1} \zeta_{\alpha}(\theta) G\left(x-\xi,(t-\eta)^{\alpha} \theta\right) d \xi d \theta d \eta d t . \\
+\frac{\alpha L \delta}{h} \int_{0}^{h} \int_{0}^{t} \int_{0}^{\infty} \int_{R^{n}} \theta(t-\eta)^{\alpha-1} \zeta_{\alpha}(\theta) G\left(x-\xi,(t-\eta)^{\alpha} \theta\right) d \xi d \theta d \eta d t \\
\leq \frac{L h^{\alpha}\left|\alpha_{1}(x)\right|}{\alpha+1}+\frac{L \delta h^{\alpha}}{\alpha+1}=\frac{L \delta h^{\alpha}}{(\alpha+1)-L h^{\alpha}} \equiv G_{1},
\end{gathered}
$$


Then

$$
\left|\alpha_{2}(x)\right| \leq G_{1} \equiv \frac{L \delta h^{\alpha}}{(\alpha+1)-L h^{\alpha}}=\delta \frac{\varepsilon-L h^{\alpha}}{1+L h^{\alpha}} \equiv G_{\varepsilon}
$$

Thus from (1.10) and using (1.16), (1.23) and (1.25) we get

$$
\begin{aligned}
\left|\delta_{2}(x, t)\right| \leq & L \alpha\left|u_{1}(x, t)+\alpha_{2}(x)-\alpha_{1}(x)\right| \int_{0}^{t}(t-\eta)^{\alpha-1} d \eta \\
\leq & L h^{\alpha}\left\{\delta+\left|\alpha_{2}(x)\right|\right\}=L h^{\alpha} \delta\left\{1+\frac{L h^{\alpha}}{(\alpha+1)-L h^{\alpha}}\right\} \\
& \left|\delta_{2}(x, t)\right| \leq \frac{L h^{\alpha} \delta(\alpha+1)}{(\alpha+1)-L h^{\alpha}} \equiv G_{2}
\end{aligned}
$$

and so

$$
\left|\delta_{2}(x, t)\right|+\left|\alpha_{2}(x)\right| \leq \frac{L h^{\alpha} \delta(\alpha+1)}{(\alpha+1)-L h^{\alpha}}+\frac{L h^{\alpha} \delta}{(\alpha+1)-L h^{\alpha}}=\frac{L h^{\alpha} \delta(\alpha+2)}{(\alpha+1)-L h^{\alpha}}=\delta \varepsilon
$$

From (1.15) at $\mathrm{m}=3$ and (1.16) we get

$$
\left|\alpha_{3}(x)\right| \leq \frac{\alpha L}{h} \int_{0}^{h} \int_{0}^{t}(t-\eta)^{\alpha-1}\left|\delta_{2}(x, t)+\alpha_{3}(x)-\alpha_{2}(x)\right| d \eta d t
$$

From the inequality (1.26), we get the estimation

$$
\left|\alpha_{3}(x)\right| \leq \frac{L h^{\alpha} \delta}{(\alpha+1)-L h^{\alpha}} \varepsilon=G_{1} \varepsilon .
$$

Then from (1.14) at $\mathrm{n}=3$,

$$
\begin{gathered}
\left|\delta_{3}(x, t)\right| \\
\leq \alpha L \int_{0}^{t} \int_{0}^{\infty} \int_{R^{n}} \theta(t-\eta)^{\alpha-1} \zeta_{\alpha}(\theta) G\left(x-\xi,(t-\eta)^{\alpha} \theta\right)\left|u_{2}(x, t)+\alpha_{3}-u_{1}-\alpha_{2}\right| d \xi d \theta d \eta \\
\leq L h^{\alpha}\left\{\delta \varepsilon+\frac{L h^{\alpha} \delta \varepsilon}{(\alpha+1)-L h^{\alpha}}\right\}=\frac{L h^{\alpha} \delta \varepsilon(\alpha+1)}{(\alpha+1)-L h^{\alpha}}=G_{2} \varepsilon
\end{gathered}
$$

In general, if $\left|\alpha_{m-1}(x)\right| \leq G_{1} \varepsilon^{m-3}$,

$$
\begin{gathered}
\left|\delta_{m-1}(x, t)\right| \leq G_{2} \varepsilon^{m-3} \text {, then } \\
\left|\delta_{m-1}(x, t)\right|+\left|\alpha_{m-1}(x)\right| \leq \delta \varepsilon^{m-2},
\end{gathered}
$$

and from (1.15), we get

$$
\left|\alpha_{m}(x)\right| \leq \frac{\alpha L}{h} \int_{0}^{h} \int_{0}^{t}(t-\eta)^{\alpha-1}\left|\delta_{m-1}(x, t)+\alpha_{m}(x)-\alpha_{m-1}(x)\right| d \eta d t
$$


Then $\left|\alpha_{m}(x)\right| \leq G_{1} \varepsilon^{m-2}$, and so from formula (1.14) we get $\left|\delta_{m}(x)\right| \leq G_{2} \epsilon^{m-2}$ Consequently, from the inequality (1.24), when $m \rightarrow \infty, u_{m}(x, t)$ on the interval $[0, a]$ uniformly converges to $u(x, t)$, which satisfy $(1.4)$.

\section{Theorem 1.3:}

Suppose $0<\alpha<1, f(x, t, u(x, t))$ satisfies the conditions of theorem (1.2) and also (1.17) is satisfied. Then $u_{m}(x, t)$ which constructed by the previous method is approximated by $u(x, t)$ on the interval $[0, a]$ and so the following inequality is satisfied

$$
\begin{gathered}
\left|u(x, t)-u_{m}(x, t)\right| \leq \frac{\delta \eta}{1-L h^{\alpha}}\left\{1-\frac{2 L h^{\alpha}}{1+L h^{\alpha}}\left(1-\eta^{m-1}\right)\right\} \varepsilon^{m}, \\
\eta=(\alpha+1)-\frac{L h^{\alpha}}{\alpha+2}
\end{gathered}
$$

proof:

Using (1.4), (1.5) and the inequality (1.16), we get

$$
\begin{gathered}
\left|u-u_{1}\right| \\
=\alpha \int_{0}^{t} \int_{0}^{\infty} \int_{R^{n}} \theta(t-\eta)^{\alpha-1} \zeta_{\alpha}(\theta) G\left(x-\xi,(t-\eta)^{\alpha} \theta\right)\left|f(\xi, \eta, u(\xi, \eta))-f\left(\xi, \eta, \alpha_{1}(\xi)\right)\right| d \xi d \theta d \eta \\
\leq \alpha L \int_{0}^{t}(t-\eta)^{\alpha-1}\left\{\left|u-u_{1}\right|+\left|u_{1}-\alpha_{1}\right|\right\} d t \\
\leq \frac{\delta L h^{\alpha}}{1-L h^{\alpha}} \equiv G_{3} .
\end{gathered}
$$

From (1.1), (1.8) and the inequality (1.16) we can deduce

$$
\begin{gathered}
\left|u-u_{2}\right| \\
=\alpha \int_{0}^{t} \int_{0}^{\infty} \int_{R^{n}} \theta(t-\eta)^{\alpha-1} \xi_{\alpha}(\theta) G\left(x-\xi,(t-\eta)^{\alpha} \theta\right)\left|f(\xi, \eta, u(\xi, \eta))-f\left(\xi, \eta, u_{1}(\xi, \eta)+\alpha_{2}\right)\right| d \xi d \theta d \eta \\
\leq \alpha L \int_{0}^{t}(t-\eta)^{\alpha-1}\left\{\left|u-u_{1}\right|+\left|\alpha_{2}\right|\right\} d \eta .
\end{gathered}
$$

and then from (1.25) and (1.27)

$$
\begin{gathered}
\left|u-u_{2}\right| \leq\left(G_{3}+G_{\varepsilon}\right) L h^{\alpha}=\frac{\delta L h^{\alpha}}{1+L h^{\alpha}}\left(1+\frac{2 L h^{\alpha}}{1-L h^{\alpha}} \eta\right) \varepsilon, \\
\eta=\frac{L h^{\alpha}}{\varepsilon}=\frac{(\alpha+1)-L h^{\alpha}}{\alpha+2}
\end{gathered}
$$


According to (1.1), formula (1.12) and the inequality (1.6) we can write

$$
\begin{aligned}
\left|u-u_{m}\right| & \leq \alpha L \int_{0}^{t}(t-\eta)^{\alpha-1}\left|u-u_{m-1}-\alpha_{m}\right| d \eta \\
& \leq L h^{\alpha}\left(\left|u-u_{m-1}\right|+\left|\alpha_{m}\right|\right) .
\end{aligned}
$$

From (1.20) and the inequality

$$
\left|u-u_{m-1}\right| \leq \frac{\delta L h^{\alpha}}{1+L h^{\alpha}} \quad\left(1+\frac{2 L h^{\alpha}}{1-L h^{\alpha}} \eta^{m-2}\right) \varepsilon .
$$

we get

$$
\begin{aligned}
\left|u-u_{m}\right| & \leq \frac{\delta L h^{\alpha}}{1+L h^{\alpha}}\left(1+\frac{2 L h^{\alpha}}{1-L h^{\alpha}} \eta^{m-1}\right) \varepsilon^{m-1} \\
& =\frac{\delta \eta}{1-L h^{\alpha}}\left(1-\frac{2 L h^{\alpha}}{1+L h^{\alpha}}\left(1-\eta^{m-1}\right)\right) \varepsilon^{m},(\sec [7]) .
\end{aligned}
$$

Hence the required result.

\section{References}

[1] Podlubny,I.Fractional differential equation.Acad. Press,San Diego-New York-London(1999).

[2] Samko,S.G,Kilbas,A.And Marichev,O.I,Integrals and derivatives of fractional order and some of applications.

[3] EL-Borai M.M., Some probability densities and fundamental solutions of fractional evolution equation, Chaos Solitons §Fractals 14(2002) 433-440.

[4] Abujabal HAS,EL-Borai M.M..On the Cauchy problem for some abstract nonlinear differential equation.Korean J Comp. Appl Math 1996,3(7).

[5]EL-Borai M.M.,Evolution equations without semigroup,Applied mathematics and computation 149(2004)815-821.

[6] S.A.MARZAN,An approximate solution of the Cauchy problem with Caputo fractional derivative of order $0 \prec \alpha \prec 1$. The Journal of national academic science bellroussi No 1(2005) 28-34. 
[7] Gorenflo R,Mainardi F,Fractional calculus and stable probability distributions.Arch Mach 50(1995).

Received: May 30, 2007 